\section{Michele Prandi}

Université de Gênes

Italie

(iD https://orcid.org/0000-0002-6223-6946

\section{L'identification des arguments et la hiérarchisation des marges : critères formels et critères conceptuels}

Identifying arguments and hierarchizing margins: formal and conceptual criteria

\begin{abstract}
The distinction between arguments and margins within a simple nuclear sentence is sharp at conceptual level in that it is grounded in explicit relevance criteria: arguments are saturated referential noun phrases that are essential for the integrity of the process; different layers of margins enrich different kinds of processes according to different consistency requirements. If one observes the syntactic structure of linguistic expressions, on the other hand, the same distinction seems to shade into a sort of continuum owing to two orders of factors. First, there is a cleavage between the model sentence, whose main function should be the expression of the process, and the utterances actually documented in texts and corpora, whose structure is shaped by the incommensurate function to adapt the structure of the process to the communicative dynamism of a text. Moreover, within the model sentence itself, the coding regime of arguments and the coding regime of margins shadow into one another: some margins are coded, like arguments, through formal grammatical relations, while some arguments are coded, like margins, directly as conceptual relations through a set of forms of expression motivated by their conceptual content.

In spite of these obstacles, the conceptual distinction between arguments and margins and the hierarchy of margins can be identified at the level of model sentence thanks to adequate and differentiated criteria. These criteria are formal where the difference of coding regime draws a sharp formal distinction between arguments and margins, and conceptual and textual where the structure of the forms of expression neutralises the distinction. Conceptual and textual criteria also make the identification of a clear hierarchy of margins possible.
\end{abstract}

\title{
Keywords
}

Arguments, inner margins, outer margins, formal criteria, conceptual and textual criteria, control, closeness, coding regime 
La structure syntaxique du noyau d'une phrase simple et la structure de son signifié — du procès (L. Tesnière, 1965 [1959]) — se présentent comme autant de réseaux de relation entre constituants. Une relation comporte toujours un terme actif, qui institue et contrôle la structure, et un ou plusieurs termes passifs, les arguments. Dans une phrase comme Marguerite regarde la lune, par exemple, le verbe regarder institue une relation qui entraîne deux arguments : Marguerite et la lune. Dans une phrase comme La lune brille, le verbe briller institue une relation qui entraîne un seul argument : la lune. Les termes qui instituent des relations sont les verbes, comme briller ou admirer, les adjectifs, comme triste ou jeune, et les noms relationnels, comme conseil ou départ. Les termes qui fournissent les arguments sont des noms propres, comme Jean ou Marguerite, des pronoms, comme elle ou nous, ou des syntagmes nominaux, comme la lune ou notre village.

En tant que pivots de relations, les termes relationnels sont des termes non saturés (L. Tesnière, 1965 [1959]), et ne peuvent assurer leur fonction que s'ils sont complétés par un nombre adéquat d'arguments. Sur la base de cette prémisse, la question centrale pour l'analyse de la structure syntaxique de la phrase modèle et de son signifié complexe consiste à identifier, pour chaque pivot prédicatif, et notamment pour chaque verbe ${ }^{1}$, les arguments dont il a besoin pour former une phrase capable de construire un procès intègre, et à les distinguer des déterminations non argumentales, ou marges ${ }^{2}$. En deuxième instance, la distinction principale entre arguments et marges doit être complétée, d'une part par une hiérarchisation des marges, et d'autre part par l'identification des constituants qui, tout en étant essentiels pour l'intégrité de certains procès, ne sont pas des arguments. La hiérarchie des marges inclut, à côté des circonstanciels identifiés par Tesnière, les marges du prédicat et les modificateurs du verbe. Les constituants essentiels du procès incluent, à côté des arguments, certains adverbes et les attributs du sujet et de l'objet direct requis par certains verbes.

Dans ma contribution, j'aborderai dans un premier temps les questions interconnectées de la distinction entre arguments et marges et de la hiérarchisation des marges dans la phrase modèle. Étant donné le décalage entre la phrase modèle et l'énoncé d'une part, et les conditions de codage différenciées des arguments dans la phrase modèle d'autre part ( $(1.1)$, les critères qui nous permettent d'aborder

${ }^{1}$ En présence de verbes polysémiques, chaque acception présente en principe sa valence. Les variations peuvent affecter le nombre des arguments et leur forme. Le verbe céder, par exemple, a une acception intransitive monovalente - Le mur a cédé -, une acception intransitive divalente - Jean a cédé à la requête de Pierre - et un emploi transitif trivalent : L'enfant a cédé sa place au grand-père. À parité de nombre d'arguments, compter est transitif dans une acception - Marc est en train de compter les éleves - et intransitif dans l'autre : Martine compte sur ton aide.

${ }^{2}$ L'étiquette marge (R. E. Longacre, 2006 [1985]) est préférable à celle traditionnelle de circonstanciel du fait qu'elle permet de différencier des cuches différentes, et notamment les marges externes du procès, qui sont des circonstanciels, et les marges du prédicat. 
la double question ne peuvent qu'être à la fois indirects et différenciés. Dans le noyau de la phrase, l'identification des relations grammaticales à l'aide de critères formels implique l'identification des arguments associés, à une exception près $(\S 2.2)$. Les critères formels sont inutilisables, par contre, pour identifier un groupe d'arguments qui partagent avec les marges le codage direct et motivé de la forme d'expression. Pour identifier ces arguments et les distinguer des marges de la même forme, nous sommes obligés à avoir recours à des critères conceptuels, qui visent immédiatement la cohérence des relations et déplacent l'analyse dans une dimension textuelle. Les mêmes critères conceptuels et textuels assurent la hiérarchisation des marges (§ 2.3).

Après avoir analysé les adverbes et les attributs qui, tout en donnant une contribution essentielle à l'intégrité de certains procès, ne sont pas des arguments (§ 3), je discuterai l'hypothèse, avancée par quelques linguistes, selon laquelle la présence de marges internes au procès d'une part, et de constituants essentiels non argumentaux d'autre part, serait à interpréter comme la preuve que les arguments et les marges identifient les deux pôles d'une opposition graduée à l'intérieur d'un continuum (\$ 4). Cette idée a pris de l'essor dans les dernières années, poussée par les analyses guidées sur corpus, ou corpus driven, qui déplacent l'objet d'étude de la phrase modèle aux énoncés documentés dans les corpus.

\section{La valence des verbes : structure conceptuelle, énoncé, phrase modèle}

Tant la distinction entre arguments et marges que la distinction entre différents couches de marges, et notamment entre marges du procès, marges du prédicat et modificateurs du verbe (M. Prandi, 2004 : 270 - 276), sont des différences de nature conceptuelle, fondées sur les critères de l'intégrité et de la cohérence des procès.

D'une part, l'intégrité d'un procès demande la présence d'un nombre donné d'arguments avec un profil conceptuel donné : un procès comme «bailler », par exemple, ne demande qu'un argument : l'expérienceur ; " admirer » en demande deux : l'expérienceur et le stimulus ; « raconter» en demande trois : le locuteur, le destinataire et le message.

D'autre part, l'accessibilité des marges aux différents types de procès est soumise à des conditions de cohérence claires et observables, qui imposent la distinction entre différentes couches. En tant que marges externes, les circonstances spatiales et temporelles sont compatibles avec tout procès actualisé. Les marges du prédicat internes au procès comme l'instrument, le collaborateur de l'agent ou le bénéficiaire, ne sont cohérentes que si elles sont associées à une action. 
Les modificateurs, par contre, sont sélectionnés directement par chaque verbe en fonction de leur cohérence avec le contenu spécifique du procès : tout événement peut se passer soudainement; seulement une action peut être exécutée avec soin ; seulement un acte de parole peut être accompli à voix haute.

Le premier critère impose sur le plan logique la distinction entre arguments et marges : les marges s'opposent en bloc aux arguments du fait que leur fonction ne consiste pas à garantir l'intégrité du procès mais à enrichir un procès intègre. Toujours sur le plan logique, le second critère impose la distinction entre marges externes de procès génériques, marges internes de prédicats d'action et modificateurs du verbe. Si cela est vrai, les deux ordres de distinctions s'imposent comme incontournables. Au moment d'aborder la description linguistique, cependant, la question logique fait place à un certain nombre de questions empiriques qui, d'une certaine façon, en offusquent la clarté : y-a-t-il des critères explicites qui permettent de reconnaitre la double distinction dans la structure syntaxique ou dans le contenu des expressions ? Si la réponse est positive, à quelles conditions ? S'il y a des difficultés, comment les affronter ou les évaluer ?

L'identification des arguments d'un verbe donné et la hiérarchisation des marges ne sauraient être immédiates et directes qu'à une condition : que la structure syntaxique de la phrase reflète, comme un miroir, la structure conceptuelle d'un procès. Or, à la différence des formules du calcul des prédicats de forme Px, Pxy, Pxyz, qui contiennent tous et seuls les arguments d'un pivot prédicatif d'un prédicat au sens logique, ou prédicateur ${ }^{3}$ - les expressions linguistiques ne sont pas l'épiphanie d'un schéma d'arguments, mais répondent à des fonctions différentes et stratifiées qui modèlent leur forme. La spécificité des expressions linguistiques et leur distance des schémas d'arguments se manifeste à deux niveaux : dans le décalage entre la phrase modèle et les énoncés documentés dans les textes et dans les corpus d'une part; dans les conditions de codage de la structure de la phrase modèle d'autre part.

\subsection{L'énoncé}

Dans les textes et dans les corpus, nous ne trouvons pas des phrases modèles mais des énoncés, et donc des équivalents fonctionnels de phrases qui opèrent dans un régime indexical comme signaux de messages contingents (M. Prandi, 2019). La fonction d'un énoncé consiste à insérer le procès qu'il hérite de la phrase modèle dans un texte ou dans un acte de parole de façon cohérente avec la progression de son dynamisme communicatif (J. Firbas, 1964). La fonction textuelle (M. A. K. Halliday, 1970) affecte la forme de l'expression à deux niveaux. En

${ }^{3}$ Le terme prédicateur (predicator) est utilisé par Lyons (1977 : 434) pour distinguer le pivot relationnel d'une prédication de la relation grammaticale de prédicat, contrepartie du sujet. 
premier lieu, la structure de la phrase modèle subit des manipulations qui sont fonctionnelles à la perspective communicative requise par son environnement textuel ou discursif : un énoncé comme Ce livre, Marc l'a acheté à la gare, par exemple, se différencie de la phrase modèle - Marc a acheté ce livre à la gare - du fait que l'objet direct, au référent duquel la progression du dynamisme communicatif confère la fonction de thème, est disloqué en première position. Ensuite, et surtout, le même mécanisme d'adaptation porte à une distribution de constituants dans l'énoncé qui reflète leur contribution au dynamisme communicatif sans nécessairement garantir l'intégrité du procès. Si nous mesurons la structure des énoncés à partir du schéma d'arguments que la phrase modèle a la fonction de mettre en place, nous constatons un double décalage. D'une part, certains arguments qui sont requis pour l'intégrité d'un procès mais dont le poids communicatif est négligeable ne sont pas spécifiés. Dans un énoncé comme Paul a vendu son vélo, par exemple, on remarque l'absence d'un argument - le destinataire — qui est essentiel à l'intégrité de l'action. D'autre part, un énoncé peut contenir des expressions qui, tout en n'étant pas des arguments, donnent une contribution essentielle au dynamisme communicatif, et notamment des circonstances, des marges du prédicat et des modificateurs du verbe. Un énoncé comme Dans ce magasin, on achète bon marché ignore à la fois la marchandise et la source mais spécifie les circonstances spatiales et la manière. En termes de dynamisme communicatif, l'énoncé Paul a vendu son vélo s'insère naturellement dans un environnement communicatif où l'identification de l'acheteur n'est pas pertinente ; l'énoncé Dans ce magasin, on achète bon marché s'adapte à un contexte où le lieu et la manière sont plus saillants que la source et la marchandise.

Lors d'une analyse guidée sur des corpus, il est essentiel de ne pas confondre de telles configurations, dont la pertinence est communicative, avec la documentation d'une variabilité des schémas d'arguments liée à la polysémie d'un bon nombre de verbes. Si la différence de configuration entre deux énoncés comme Paul a vendu son vélo et Paul a vendu son vélo à Louis cache un seul et même schéma d'arguments, la différence entre Jean a lu et Jean a lu "Les Misérables » renvoie à deux schémas d'arguments différents ${ }^{4}$. Comme « le corpus ne contient pas en lui-même les conditions de possibilité de sa propre connaissance » (A. Orlandi, M. Fasciolo, 2021) et, plus généralement, comme l'expérience ne fournit pas les catégories nécessaires pour sa description, les données documentées par les énoncés attestés dans un corpus doivent être évaluées au jour de la phrase modèle dont chaque énoncé est l'équivalent fonctionnel's.

${ }^{4}$ L'emploi intransitif de verbes comme manger ou lire se distingue de l'emploi transitif du fait que l'objet interne qu'il incorpore n'identifie pas un référent spécifique : Je l'ai lu signifie 'J'ai lu le livre particulier que tu connais' ; J'ai lu signifie 'J'ai accompli un acte de lecture'.

${ }^{5}$ La phrase « n'est pas une donnée empirique brute mais une donnée éidétique - un modèle qui confère aux données brutes de l'expérience leur structure et leur valeur. Il faut souligner que les objets éidétiques ont la même réalité, dans leur ordre, que les données empiriques brutes, dans 


\subsection{La phrase modèle}

La phrase modèle est une phrase nucléaire qui, à côté d'un prédicateur, et notamment d'un verbe, contient toutes et seules les structures fonctionnelles à l'idéation d'un procès ${ }^{6}$. Sa structure inclut un noyau formé par le verbe et la configuration maximale de ses arguments, complétée, quand il est requis, par un modificateur ou un attribut. L'intégrité d'un procès comme « frapper» dans l'acception concrète, par exemple, requiert deux arguments, un agent ou une force, et un patient : par exemple Jean a frappé le serpent, La foudre a frappé l'aiguille $d u$ clocher. L'intégrité d'un procès comme Cette nouvelle a rendu Jeanne furieuse demande, à côté de deux arguments, un attribut appliqué à l'objet direct. Une fois que le verbe est saturé et qu'un noyau de procès est construit, il est prêt à recevoir des marges dont la spécification répond à un critère de cohérence conceptuelle. Si les circonstances externes sont compatibles avec tout procès actualisé, les marges internes imposent des condition de cohérence plus restrictives accessibles à la description empirique. À la différence d'un événement comme La foudre a frappé l'aiguille du clocher, qui n'admet que des circonstances externes, une action comme Jean a frappé le serpent admet d'être enrichie de façon cohérente par un instrument, un but, un bénéficiaire, et d'autres marges internes. Si l'analyse proposée est correcte, une première question pertinente pour la description de la phrase modèle est double : il s'agit d'une part de discriminer entre arguments et marges, et d'autre part de hiérarchiser les marges cohérentes, à l'aide de critères explicites (§ 2). Nous aborderons la question des adverbes et des attributs essentiels dans une section successive (§3).

Le critère le plus immédiat pour identifier les arguments - le critère de la spécification obligatoire - n'est pas utilisable, nous l'avons vu, à cause du décalage entre la phrase modèle et l'énoncé. Le fait qu'un constituant est essentiel pour l'intégrité du procès n'implique pas que sa spécification soit obligatoire dans l'énoncé. Les critères disponibles, de ce fait, sont tous indirects.

Un premier critère, entièrement conceptuel et basé sur l'intuition des locuteurs, est la latence. Si un argument essentiel pour l'intégrité d'un procès n'est pas spécifié dans un énoncé, on perçoit en principe une latence : dans un énoncé comme Marc a raconté un tas de blagues, par exemple, nous percevons immédiatement la latence du destinataire. L'évaluation de la latence, cependant, n'est pas toujours si immédiate. Dans un énoncé comme Jean a vendu son vélo à Pierre, nous percevons une sorte de latence de la somme payée du fait que ce rôle est encapsulé dans le signifié relationnel de vendre. Cependant, le critère de l'encapsulation lexicale, s'il justifie la latence, n'est pas suffisant pour conclure au statut

la mesure où ils sont également partagés par les locuteurs : parmi les objets des sciences humaines, 'être' équivaut à 'être partagé' » (M. Prandi, 2019 : 135).

${ }^{6}$ La structure de la phrase modèle nucléaire est purifiée, pour ainsi dire, de toutes les formes justifiées par les fonctions interpersonnelle et textuelle-communicative (M. A. K. Halliday, 1970). 
d'argument ${ }^{7}$, qui doit être justifié par des critères indépendants. Un verbe comme téléphoner, par exemple, encapsule l'instrument, qui n'est pas un argument mais une marge du prédicat (M. Prandi, 2004 : 272-274). Un verbe comme chuchoter, également, encapsule un modificateur, qui ne satisfait même pas les conditions préliminaires pour être un argument (§3).

Les critères formels, basés sur les propriétés grammaticales observables dans l'expression, s'interrogent sur la relation entre la forme des constituants de la structure syntaxique d'une phrase et le rôle joué par leurs référents dans la structure du procès : l'hypothèse est que la forme d'expression des arguments présente des marques formelles spécifiques capables de la différencier de la forme d'expression des marges. Étant donné que la structure syntaxique de chaque phrase contient un noyau formé par des relations grammaticales formelles entouré par différentes couches de formes d'expressions dont la structure est motivée par le contenu des relations conceptuelles exprimées, une hypothèse raisonnable est qu'un tel décalage dans le régime de codage soit corrélé à la distinction entre arguments et marges. L'observation des données, cependant, nous montre qu'une telle affinité élective entre formes et fonctions, tout en étant significative, n'est pas systématique. D'une part, en présence de certains verbes et de certaines constructions, certains rôles marginaux sont codés par l'intermédiaire d'une relation grammaticale formelle ; d'autre part, certains arguments sont codés par des formes d'expression dont la structure grammaticale, comme la structure des marges, n'est pas autonome mais motivée par le contenu conceptuel. Cette circonstance nous oblige à identifier des critères de discrimination supplémentaires et différenciés, spécifiques pour chaque typologie de relation entre rôles et formes de codage.

Dans la suite de ce paragraphe, je vais mettre en relief en premier lieu les différences pertinentes entre les deux régimes de codage actifs dans la structure de la phrase, à savoir, le codage relationnel, fondé sur la présence de relations grammaticales formelles, et le codage ponctuel, basé sur la structure interne de la forme d'expression ( $\$ 2.1$ ). Ensuite, je vais examiner deux cas de manque de corrélation entre régime de codage et fonction : une construction dans laquelle une relation grammaticale, l'objet indirect, est associée non pas à un argument mais à un éventail de relations conceptuelles marginales (§ 2.2), et des cas significatifs d'arguments codés sans le support de relations grammaticales $(\S 2.3)$.

${ }^{7}$ Les arguments incorporés dans le signifié lexical d'un verbe, comme le rêve dans rêver, sont appelés shadow arguments, 'arguments ombre', par Pustejowsky $(1995,2000)$. 


\section{Le codage des arguments et des marges dans la phrase modèle}

\subsection{Codage relationnel et codage ponctuel}

La différence entre le codage relationnel et le codage ponctuel est une donnée empirique observable dans la structure de la phrase : elle dépend de la présence ou de l'absence de relations grammaticales autonomes qui médiatisent la relation entre un constituant et un rôle ${ }^{8}$. Dans une phrase comme Mon fils a coupé le bois avec une hache, par exemple, chaque expression nominale et prépositionnelle finit par coder un rôle du procès, mais pas aux mêmes conditions. La relation entre le syntagme avec une hache et l'instrument est directe. La relation entre mon fils et l'agent et entre le bois et le patient, par contre, n'est pas directe, mais se fait par l'intermédiaire d'une relation grammaticale, respectivement le sujet et l'objet direct. Cette différence n'est que le segment visible d'une différence multifactorielle. Les relations grammaticales sont des catégories formelles et relationnelles qui ne focalisent pas la structure interne de chaque constituant mais sa relation avec la structure hiérarchique de la phrase. En français, et plus généralement dans les langues dépourvues de cas, le sujet et l'objet direct ont exactement la même forme, mais entretiennent des relations différentes avec la structure qui les accueille : le sujet est un constituant immédiat de la phrase, alors que l'objet direct est un constituant du prédicat. Dans ces conditions, l'association d'expressions et de rôles n'est pas seulement indirecte, mais aussi globale : l'activation d'un rôle engage non pas l'expression isolée, mais la construction comme hiérarchie de relations et le procès comme hiérarchie de rôles. C'est la raison qui m'a poussé à parler de codage relationnel.

À la différence des arguments codés en régime relationnel, les rôles marginaux codés en régime ponctuel se caractérisent en premier lieu comme autant de relations conceptuelles. L'instrument, par exemple, est défini sur la base de sa relation cohérente avec la structure conceptuelle d'une action : il s'agit d'un objet utilisé par un agent pour accomplir une action. En tant que structures conceptuelles autonomes, les rôles marginaux sont directement accessibles à la pensée cohérente - à l'inférence - indépendamment de leurs formes spécifiques de codage. Cela implique qu'ils peuvent être atteints par deux chemins indépendants, à savoir le codage linguistique et l'inférence.

En l'absence d'un contrôle verbal et de relations grammaticales indépendantes, le codage ponctuel dépend entièrement du contenu du mot de liaison, qui dans la phrase simple est une préposition. En même temps, l'accessibilité directe des structures conceptuelles autorise une prévision : le codage de la part de la

${ }^{8}$ La présence de relations grammaticales autonomes dans le noyau de la phrase est un paramètre typologique : voir Lazard (1998: 118). 
préposition n'est pas une condition nécessaire pour l'identification d'une structure conceptuelle marginale. En effet, si nous mesurons le pouvoir de codage des prépositions à partir du modèle conceptuel accessible de façon indépendante, nous constatons qu'il s'agit d'une grandeur graduée, s'étalant d'un codage insuffisant, ou sous-codage, à un codage qui active une relation plus riche que la relation accessible à l'inférence, ou sur-codage, en passant par un codage équilibré, où la préposition code exactement la relation accessible à l'inférence. En présence d'un codage insuffisant, c'est l'inférence, fondée sur l'accès direct aux concepts cohérents, qui prend le relais du codage pour accomplir la tâche inachevée.

Un exemple de codage adéquat est la préposition malgré. Dans Le cerisier est en fleur malgré la neige, la préposition code toutes les composantes conceptuelles de la relation concessive cohérente, à savoir la réalité des deux procès, la succession temporelle et l'implicite de cause frustrée.

Un exemple de codage insuffisant est la préposition avec, qui code une relation de cooccurrence asymétrique, trop pauvre pour identifier un rôle quelconque. Grâce à l'enrichissement inférentiel, l'expression prépositionnelle exprime l'instrument en (1), le collaborateur de l'agent en (2) et la manière de l'action en (3). Comme le montrent les exemples (4) et (5), en outre, elle est prête à introduire n'importe quelle relation conceptuelle marginale imaginable, à la seule condition qu'elle soit cohérente :

1. Luc a abattu le peuplier avec une scie

2. Luc a abattu le peuplier avec Marc

3. Luc a abattu le peuplier avec beaucoup de peine

4. Luc est entré dans la salle avec un beau sourire

5. Luc est entré dans la salle avec un grand chapeau

Un exemple de surcodage est l'expression de la relation finale qui utilise des noms relationnels au contenu spécifique. Une expression comme Pierre a fait des études d'informatique dans le but de changer de travail se limite a coder la relation conceptuelle de but. L'expression Pierre a fait des études d'informatique avec l'ambition de changer de travail enrichit la relation avec une nuance sémantique inséparable du signifié du nom ambition, et donc du codage linguistique (G. Gross, M. Prandi, 2004).

Les exemples examinés documentent la corrélation attendue entre codage relationnel et arguments d'une part, codage ponctuel et marges d'autre part. Dans la réalité empirique, cependant, l'affinité élective est perturbée par des ruptures de la corrélation dans les deux sens : à côté de marges associées à une relation grammaticale, nous trouvons des arguments codés en régime ponctuel. 


\subsection{Marges confiées à une relation grammaticale : l'extension de la construction ditransitive}

Les relations grammaticales qui forment le noyau d'une phrase modèle peuvent être identifiées par des critères formels, basés sur leurs propriétés grammaticales et sur leur comportement en tant que formes. En français, par exemple, le sujet grammatical s'accorde avec la forme verbale du prédicat; sa spécification est obligatoire ; le rôle qui l'occupe est marginalisé dans la phrase passive équivalente ; s'il est disloqué, il doit être repris par une forme clitique. Dans la mesure où les relations grammaticales nucléaires sont associées à des arguments, les critères formels sont censés identifier en même temps des arguments. Le sujet, par exemple, est systématiquement associé au premier argument. La corrélation entre relations grammaticales et arguments, cependant, n'est pas absolue, car il y a un cas de relation grammaticale qui ne code pas un argument : il s'agit de l'objet indirect de la construction ditransitive étendue aux verbes divalents, par exemple Jeanne a cuit une tarte à Marc. Malgré la présence de trois relations grammaticales, force est de reconnaître que seulement les deux premières - le sujet et l'objet direct - accueillent des arguments, à savoir l'agent et le patient. Le contenu de la position ouverte par l'objet indirect, au contraire, n'est pas contrôlé par le verbe, comme il arrive en présence d'arguments, mais il est le résultat d'une inférence motivée pour des raisons de cohérence conceptuelle, comme il est typique des marges. Grâce à l'inférence, l'objet indirect exprime le bénéficiaire dans une construction comme Jeanne a cuit une tarte à Marc et le possesseur externe (E. König, M. Haspelmath, 1998) dans Marie lui a lavé les cheveux ${ }^{9}$. En italien et en espagnol, l'éventail des rôles admis dans la position d'objet indirect inclut, à côté du bénéficiaire $(1,1 \mathrm{a})$ et du possesseur externe $(2,2 \mathrm{a})$, le substitut de l'agent $(3,3 \mathrm{a})$ :

1. Maria ha cucinato una torta a Paolo

1a. Mamá le preparó una paella a Luisa

2. Maria ha cucito la giacca a Piero

2a. Pablo le reparó el coche a su hermano

3. Giovanna ha cotto una torta a Maria per i suoi invitati

3a. Juana le ha cocido una tarta a María para sus invitados

Aucune des relations conceptuelles confiées à l'objet indirect dans les constructions examinées n'est un argument. En premier lieu, un argument est nécessairement un rôle. Or, parmi les relations conceptuelles activées dans nos exemples, il y en a une - le possesseur externe — qui n'est pas un rôle. Ensuite,

9 À l'avis de Vergnaud et Zubizarreta (1992: 598), l'expression du possesseur externe avec l'objet indirect en français n'est admise qu'en présence de pronoms clitiques : Une pierre lui a cassé la jambe ; ?ne pierre a cassé la jambe à Jacques. 
nous avons identifié les différentes relations conceptuelles par inférence, sur la base d'un critère de cohérence conceptuelle. Or, ce comportement est incompatible avec le statut d'argument, dont le contenu est contrôlé par le verbe. Finalement, les différentes relations conceptuelles peuvent se cumuler, toujours pour des raisons de cohérence. En (2) et (2a), par exemple, le référent de l'objet indirect cumule les rôles de bénéficiaire, de possesseur externe et de substitut de l'agent. Il s'agit, encore une fois, d'un comportement incompatible avec le statut d'argument contrôlé par le verbe. La conclusion est que l'objet indirect d'un verbe divalent comme cuire ne contient pas un argument; donc, l'extension de la construction ditransitive ne restructure pas l'action, mais se limite à l'enrichir par une relation conceptuelle marginale. Le critère crucial à cette hauteur est l'alternative entre le contrôle verbal et l'inférence dans la mise en place du contenu d'un rôle.

L'idée que le transfert de l'objet indirect change une action à deux arguments en une transaction à trois arguments naît d'une équivoque alimentée par le comportement de la construction parallèle en anglais. En anglais, l'objet indirect associé à un verbe divalent code systématiquement un seul et même rôle, le bénéficiaire. Comme Goldberg (1995 : 141) le remarque, une phrase comme Sally baked her sister a cake " can only mean that Sally baked a cake with the intention of giving it to her sister ». Cette circonstance, associée à l'idée que la construction ditransitive signifie une transaction, ce qui implique que l'objet indirect est une forme de codage ponctuel d'une famille d'arguments dont le prototype est le destinataire, et à l'idée que le bénéficiaire fait partie de cette famille au même titre qu'un destinataire, a amené Goldberg à la conclusion que l'objet indirect augmente la valence des verbes cible de la construction ditransitive et change le procès qu'ils expriment en une forme de transaction ${ }^{10}$. L'argumentation, cependant, est fallacieuse à deux niveaux.

En termes généraux, l'objet indirect n'a aucune corrélation systématique avec le destinataire d'une transaction. L'objet indirect est une relation grammaticale

${ }^{10}$ Selon Goldberg (1995: 10), « we can define the ditransitive construction to be associated directly with agent, patient, and recipient roles ». Plus généralement, "Constructions themselves carry meaning » (1995: 1). Voir aussi Hilpert (2014 : chap. 2). En fait, si une construction a un signifié ou pas est une question empirique qui dépend du régime de codage. La construction ditransitive, formée par trois relations grammaticales vides, ne peut pas avoir un signifié et est compatible avec plusieurs, en fonction du contenu du verbe : Si Jean a donné un livre à Marie dépeint une transaction, Jean envie à Marie son travail dépeint une attitude psychologique, et n'admet pas d'être interprétée comme une transaction, même métaphorique. Une construction transitive qui contient comme troisième argument une destination codée en régime ponctuel, par contre, est effectivement associée à un contenu : elle ne peut que véhiculer un déplacement dans l'espace, ou réel - Jean a transporté le vieux meuble dans le grenier - ou métaphorique : Jean a traduit ses poèmes en italien. De ce fait, l'extension de la construction de déplacement (caused motion construction) à des verbes divalents, très productive en anglais, transforme effectivement le procès cible en un déplacement : Hope [...] laughed me out of sadness (Emily Brontë : 'L'espoir me sourit hors de la tristesse') ; John blew the ant off the plate ('Jean souffla la fourmi hors du plat'). 
formelle qui reçoit dans chaque construction le rôle que le verbe contrôleur lui destine : pour ne considérer qu'un cas extrême, il contient le destinataire avec prêter et la source avec emprunter ${ }^{11}$. Comme l'objet indirect est une relation grammaticale vide, son transfert ne comporte pas le transfert d'un rôle. En termes plus spécifiques, l'assimilation du bénéficiaire au destinataire est dépourvue de fondement. Le destinataire est un argument qui qualifie une classe spécifique d'actions, à savoir les transactions, réelles comme "donner ", ou symboliques, comme " dire ». Le bénéficiaire, pour sa part, est une marge qui ne caractérise aucune action en particulier et qui est compatible avec toutes. Comme toutes les marges, le bénéficiaire enrichit une action sans changer sa structure argumentale : dans le cas particulier, l'intention de donner la tarte à quelqu'un, et donc la présence d'un bénéficiaire, ne change pas la structure de l'action de cuire, pas plus que la présence d'un possesseur ou d'un collaborateur de l'agent.

\subsection{Arguments codés en régime ponctuel}

Le codage ponctuel d'un argument est une option qui ne s'ouvre qu'en présence de formes d'expression contenant des prépositions. La réciproque, cependant, n'est pas vraie. Contrairement à une opinion partagée ${ }^{12}$, la présence d'une préposition n'est pas incompatible avec la présence d'une relation grammaticale, et donc avec le codage relationnel. Les relations grammaticales confiées à des expressions prépositionnelles sont deux, à savoir l'objet indirect (M. Prandi, 2020) et l'objet prépositionnel (R. Steinitz, 1969). Nous allons nous pencher sur la dernière relation, qui permet de mettre en relief les différences de comportement de la préposition dans les deux régimes de codage.

L'objet prépositionnel est le complément d'un verbe intransitif à deux arguments : par exemple, Marguerite compte sur ton aide. L'argument principal pour conclure que l'objet prépositionnel est une relation grammaticale vide est le comportement de la préposition. Quand elle est engagée dans l'expression d'une relation conceptuelle - par exemple d'une relation spatiale — la préposition manifeste toutes les propriétés qui qualifient le régime de codage ponctuel. Elle est choisie à l'intérieur d'un paradigme, et de ce fait elle investit son contenu dans l'expression de la relation : Le chat s'est endormi sur / sous / derrière / à côté de la table. Dans l'objet prépositionnel, la même préposition est imposée par le verbe, ne fait pas l'objet d'un choix — nous ne pouvons pas dire *Je compte au dessus de ton aide - et se vide de son contenu : elle ne signifie pas « au dessus de »

${ }^{11}$ Réciproquement, un destinataire est confié à l'objet direct par un verbe comme informer : Le professeur a informé ses élèves du changement de date (M. Prandi, 2020).

12 À l'avis de Palmer, « Marking by preposition is an indication of merely peripheral roles ». Voir aussi Lazard 1998 : 18. Gross (2012 : 135-140) souligne le double statut des prépositions, ou « indicateurs d'argument » contrôlés par un pivot prédicatif, typiquement un verbe, ou pivots de relations. 
(1969 : 41). La préposition est un instrument grammatical « incolore » (Blinkenberg, 1960) qui code une relation grammaticale vide en régime relationnel.

Si tous les syntagmes prépositionnels codés en régime ponctuel ne codaient que des marges, et que tous les arguments soient codés en régime relationnel, la distinction entre arguments et marges de forme prépositionnelle serait aisée. Mais la différence, encore une fois, n'est pas si tranchée : il y a au moins deux types d'arguments codés en régime ponctuel, dont la forme d'expression ne les distingue pas des marges au même contenu. D'une part, les expressions spatiales ont la même forme indépendamment de leur fonction de circonstanciels - Le chat s'est endormi sur / sous / derrière / à côté de / la table — ou d'arguments en présence de verbes d'état - Le chat se trouve sur / sous / derrière / à côté de / la table ou de mouvement : Le chat est allé sur / sous / derrière / à côté de / la table. D'autre part, les expressions comitatives de forme avec $+S N$ dénotant un être humain sont prêtes à exprimer tant une marge interne d'un prédicat d'action Marc a coupé le bois avec son fils - qu'un argument, et notamment l'interlocuteur, en présence de verbes de communication symétriques comme discuter: Marc a discuté le projet avec son fils. Dans tous ces cas, les critères formels sont tout à fait inefficaces, et il faut avoir recours à des critères différents, d'ordre à la fois conceptuel et textuel.

Les critères en question sont conceptuels du fait qu'ils visent à contrôler la cohérence conceptuelle d'une connexion; en même temps, ils sont textuels du fait que le but est atteint en coupant l'expression sous examen de la phrase qui exprime le noyau du procès et la déplaçant dans une phrase indépendante, pour vérifier si les deux phrases forment un texte cohérent ou pas. Or, les circonstanciels se laissent effectivement spécifier dans une phrase indépendante formée par une reprise anaphorique saturée du noyau du procès en position de sujet et par un verbe comme se passer qui prend l'expression prépositionnelle comme complément : Le chat s'est endormi. Cela s'est passé à côté de la table. En présence d'un argument de la même forme, cette reformulation est incohérente : les séquences * Le chat se trouve. Cela se passe à côté de la table et *Le chat est allé. Cela s'est passé à côté de la table ne forment pas un texte cohérent ${ }^{13}$. L'enchainement textuel a l'avantage de faire affleurer les conditions pour l'emploi cohérent des moyens de cohésion. Le pronom sujet cela est une reprise anaphorique saturée, qui n'est cohérente que si l'antécédent est un procès saturé et donc fermé à toute détermination ultérieure qui ne soit pas externe. Cela justifie tant la cohérence de la reformulation en présence d'un circonstanciel, qui s'ajoute de l'extérieur à un procès antécédent saturé, que son incohérence en présence d'un argument, qui aurait dû contribuer à le saturer à l'intérieur de la prédication. Le verbe se passer, quant à lui, prend comme sujet cohérent tout procès actualisé, et donc exactement les mêmes procès qui admettent de recevoir des circonstanciels.

${ }^{13}$ L’astérisque signale le manque de cohérence. 
À la différence d'un circonstanciel, une marge du prédicat ne se laisse pas séparer de façon cohérente par la reformulation Cela s'est passé : en effet, une reformulation comme Marc a coupé le bois. *Cela s'est passé avec son fils, quoique interprétable, n'est pas cohérente pour deux raisons. D'une part, la reprise anaphorique cela, étant saturée, traite le procès antécédent comme s'il était saturé, et donc fermé ; de ce fait, elle traite une marge interne comme si elle se situait à l'extérieur du procès. D'autre part, le verbe se passer admet comme sujet tout procès actualisé, alors qu'une marge interne n'est cohérente qu'avec une action. Pour ces mêmes deux raisons, le détachement d'une marge du prédicat demande une reprise anaphorique à la fois insaturée, et donc capable de garder ouvert le procès antécédent, et exprimant une action. Cette reprise anaphorique est le proprédicat générique d'action le faire, qui reprend le noyau du prédicat antécédent dont il hérite les marges internes. En effet, la séquence Marc a coupé le bois. Il l'a fait avec son fils forme un texte cohérent.

La reformulation avec le pro-prédicat le faire est efficace quant il s'agit de séparer les marges du prédicat, internes à une action, et les circonstanciels qui encadrent de l'extérieur tout procès actualisé. Quand il s'agit de séparer une marge interne d'un argument, par contre, la reformulation est décidément moins tranchante. Quand elle accompagne un verbe de communication symétrique comme discuter, la forme comitative exprime l'interlocuteur, qui est un argument. Malgré cela, une reformulation comme Marc a discuté le projet. Il l'a fait avec Marie ne manifeste pas son incohérence d'une façon aussi directe que, par exemple, Le chat est allé. *Cela s'est passé sous la table. La raison est probablement à chercher dans le fait que la marge du prédicat et l'argument partagent la position à l'intérieur du procès.

L'échec du test peut être surmonté grâce au critère qui oppose le contrôle verbal à l'inférence (§ 2.2). Le contenu d'un argument découle du signifié relationnel du verbe qui le contrôle, ce qui entraîne deux conséquences : l'identité du rôle est en même temps indépendant de la forme de codage et fermée à l'inférence. En présence d'une marge, tout au contraire, l'accès au contenu, qui n'est pas contrôlé par le verbe, dépend de la forme de codage et, le cas échéant, de l'inférence. Au jour de cette remarque, nous pouvons revenir au double statut de la forme comitative. Tant l'argument $(1,1 \mathrm{a})$ que la marge $(2,2 \mathrm{a})$ admettent, à côté de la forme comitative, d'entrer dans un sujet grammatical coordonné :

1. Marc a discuté le projet avec Pierre

1a. Marc et Pierre ont discuté le projet

2. Marc a coupé le bois avec Pierre

2a. Marc et Pierre ont coupé le bois

Le comportement des deux rôles, cependant, n'est pas le même dans les deux constructions. Dans le passage de (1) à (1a), le rôle de Pierre demeure inchangé : 
dans un cas comme dans l'autre, il est l'interlocuteur de Marc. La rigidité du rôle se justifie par le contrôle verbal, qui n'est pas affecté par la différence dans la forme de codage ; il s'agit donc d'un argument. Dans le passage de (2) à (2a), au contraire, le rôle de Pierre change de profil. En (2a), en position de sujet, et donc codé en régime relationnel, il reçoit son rôle par le verbe aux mêmes conditions que Marc : il ne peut qu'être le collaborateur de Marc, et donc, comme Marc, un agent de plein droit. En (2), en régime de codage ponctuel et notamment de souscodage, le statut de co-agent n'est qu'une option parmi d'autres ouverte à l'inférence. En effet, la préposition avec code la présence du réfèrent sur la scène de l'action sans lui attribuer un rôle défini ; par exemple, rien n'empêche d'imaginer un enfant admirant le dur travail de Marc. Le sous-codage laisse une marge d'indétermination que l'inférence n'est pas toujours en mesure de combler, ce qui ne peut arriver qu'en présence d'une marge.

\section{La valence au-delà des arguments : modificateurs obligatoires et attributs}

Une dernière difficulté que l'on rencontre sur le chemin d'une distinction tranchée entre arguments et marges est représentée par deux types de constituants qui sont essentiels pour l'intégrité conceptuelle de certains procès, et donc ne sont pas des marges, sans pour autant être des arguments.

En premier lieu, il y a des verbes qui ne sont pas capables de construire un procès sans la présence de certains modificateurs, que Dowty (2000:6-8) appelle "subcategorized adjuncts ». Dans des phrases comme Johnny behaved badly ou Jean s'est comporté mal, le modificateur du verbe, qui dans les constructions les plus typiques - par exemple, Jean chante mal - se comporte comme une marge, donne une contribution essentielle à l'intégrité du procès ${ }^{14}$. Cette fonction distingue radicalement ces modificateurs des modificateurs que nous pouvons percevoir comme obligatoires dans la structure de l'énoncé pour des raisons de dynamisme communicatif : par exemple, bon marché dans l'énoncé Dans ce magasin, on achète bon marché.

D'autres verbes, également, demandent comme autant de constituants essentiels pour l'intégrité du procès des attributs du sujet ou de l'objet direct (M. Riegel, J. Ch. Pellat, R. Rioul, 1994 : 234-241 ; F. Strik Lievers, 2012). Dans Jean est rentré ivre à la maison, par exemple, la présence de l'adjectif ivre, qui applique

${ }^{14}$ Comme l'écrit Dowty (2000: 7), « an adverb occurs as a complement ». Dans la terminologie que Dowty hérite de Chomsky (1965), l'adverbe sous-catégorise le verbe qu'il modifie dans le même sens où un objet direct sous-catégorise un verbe transitif. 
au sujet une prédication seconde, est fonctionnelle au dynamisme communicatif, et comme telle est une option laissée à la décision du locuteur; dans Jean semble ivre, par contre, la même prédication seconde est requise par le verbe pour l'intégrité du procès. Une prédication seconde s'applique aussi à l'objet direct de certains verbes. Le verbe rendre, par exemple, prend un attribut de l'objet comme option dans l'acception trivalente - Pierre m'a rendu le livre déchiré - et comme un constituant essentiel dans l'acception divalente : Cette nouvelle a rendu Jeanne furieuse. Dans quelques cas, la fonction de prédicat second peut être confiée à un nom — L'assemblée a élu George président — ou à un syntagme nominal : Les révolutionnaires appelaient Marat l'ami du peuple.

Ces exemples montrent que des adverbes ou des adjectifs peuvent donner une contribution essentielle à l'intégrité d'un procès, mais pas qu'ils sont des arguments. La contribution essentielle à l'intégrité du procès est une condition nécessaire pour le statut d'argument, mais pas suffisante. En effet, un argument est un actant d'un procès, et de ce fait est confié à une expression référentielle saturée. Or, ni l'adverbe, ni l'adjectif, ni même le syntagme nominal engagés dans une prédication seconde ne satisfont ces conditions. En tant que modificateur d'un verbe, qui est un terme non saturé, un adverbe est une expression non saturée au deuxième degré ; à plus forte raison, il est incapable d'introduire dans le procès un référent. Un attribut est ou un adjectif ou un nom. S'il est un adjectif, il n'est ni saturé ni engagé dans l'identification d'un référent. S'il est un nom ou un syntagme nominal, sa fonction n'est pas référentielle mais prédicative, et s'applique à un argument, et donc à un référent.

\section{Arguments et marges : deux pôles dans un continuum ?}

Nous avons constaté que l'analyse des schémas d'arguments se heurte à deux ordres de difficultés. D'une part, la distinction entre arguments et marges n'est pas directement reflétée dans la structure des énoncés. D'autre part, il y a des constituants qui échappent à une distinction tranchée entre arguments et marges du fait qu'ils partagent quelques propriétés des uns et quelques propriétés des autres. La première difficulté a amené certaines linguistes à remplacer l'idée de valence par la pure et simple documentation de la fréquence de configurations données dans les corpus ; la deuxième à encouragé l'hypothèse que les arguments et les marges ne sont que les pôles d'un continuum qui inclut toute sorte de structures hybrides.

La première difficulté, nous l'avons vu, peut être maîtrisée au niveau de la phrase modèle avec des critères adéquats. Si la question de la valence est abordée au niveau de l'énoncé, par contre, elle est insurmontable. Si l'énoncé remplace la phrase modèle comme objet même de la description, il est inévitable que 
les hiérarchies motivées par le dynamisme communicatif cachent les hiérarchies motivées par la condition d'intégrité du procès et amènent à la dissolution pure et simple de l'idée de valence. C'est précisément ce qui arrive dans les approches guidées sur corpus, qui identifient les schémas d'arguments sur la base de la fréquence et de la saillance de certaines configurations (patterns) documentée dans les corpus (P. Hanks, 2013 ; F. Perek, 2015 ; L. Mereu, 2017 ; L. Mereu, V. Piunno, 2019). L'observation des corpus, par exemple, montre qu'un verbe comme vendre n'est que rarement accompagné par tous les arguments requis pour l'intégrité du procès. Se basant sur une analyse de corpus de l'italien, par exemple, Mereu (2020 : 123 - 127) montre que la configuration la plus fréquente n'associe au verbe vendere, 'vendre', que le sujet et l'objet direct. Si les configurations documentées dans l'énoncé et leur fréquence trouvent en elles-mêmes leur critère de pertinence (F. Perek, 2015 : 28-29), « il n'y a pas quelque chose comme le nombre d'arguments de vendre », " mais des compléments accompagnant le verbe avec une fréquence majeure ou mineure » (A. Orlandi, M. Fasciolo, 2021).

Les configurations documentées dans les énoncés et leur fréquence ne sont pas immédiatement révélatrices des schémas d'arguments ${ }^{15}$ du fait qu'elles se justifient pour des raisons de dynamisme communicatif excentriques par rapport au critère de l'intégrité du procès. Si l'analyse se déplace au niveau de la phrase modèle, par contre, l'utilisation de critères adéquats, formulés en fonction des caractéristiques du problème spécifique qui, à chaque passage, offusque la clarté des distinctions pertinentes, permet de différencier les arguments tant des constituants essentiels non argumentaux que des marges, et de hiérarchiser les marges avec un degré de certitude raisonnable.

La deuxième difficulté - la présence de constituants qui partagent quelques propriétés des arguments et quelques propriétés des marges - encourage la conclusion que les arguments et les marges se réduisent aux pôles d'une opposition graduée qui encadrent un continuum. Comme l'écrit Mereu (2010 : 75), « gli argomenti e gli aggiunti non sono categorie opposte, ma scalari »; "Argomenti e margini formano un continuum nel quale i due elementi sono gli estremi di una linea che prevede in mezzo categorie che condividono in parte le proprietà dell'uno e in parte quelle dell'altro estremo » (2010: 73). Cette conclusion est l'issue d'une illusion optique qui se produit typiquement en présence de phénomènes complexes dont la structure est multifactorielle. Si nous ignorons que plusieurs facteurs indépendant peuvent interagir dans la définition d'une structure, les phénomènes caractérisés par la superposition des facteurs émergent avec un profil bien défini, alors que les phénomènes caractérisés par une dissociation présentent un profil flou, voire hybride. Dans la structure de la phrase, notamment, nous

${ }^{15}$ Un problème semblable se pose dans la perception visuelle : « au niveau de la fréquence, nous voyons plus de personnes habillées que nues ; pourtant, nous n'en concluons ni que (prototypiquement) les habits sont des parties du corps, ni que la distinction entre corps et habits est une question de continuum » (A. Orlandi, M. Fasciolo, 2020). 
identifions ainsi une opposition relativement claire entre les arguments associés aux relations grammaticales et les circonstanciels, qui sont des marges externes au procès et codées en régime ponctuel. Tous les constituants caractérisés par une dissociation de facteurs, par contre, finissent dans une zone grise qui inclut trois catégories de constituants : les arguments codés, comme des marges, en régime ponctuel ; les marges des prédicats d'action et les modificateurs du verbe qui, comme les arguments, se situent à l'intérieur du procès ; les adverbes et les attributs qui, tout en n'étant pas des arguments, sont tout aussi essentiels à l'intégrité du procès.

Pour rétablir pour chaque catégorie un profil clair et univoque et dissoudre ainsi l'idée d'un continuum, il suffit de reconnaître que la distinction entre les arguments et les marges d'une part, les arguments, les adverbes essentiels et les attributs d'autre part, résulte de l'interaction de trois paramètres, à savoir le contrôle verbal, le degré de proximité (closeness : M. Prandi, 2004) de chaque constituant du procès au verbe, et le régime de codage. Le paramètre du contrôle verbal, fondé sur le critère de l'intégrité conceptuelle du procès, justifie une opposition exclusive qui sépare les marges de tous les constituants essentiels à l'intégrité du procès sur le plan conceptuel, à savoir des arguments, des adverbes essentiels et des attributs. À l'intérieur d'un continuum gradué qui s'étend du pivot prédicatif jusqu'à la périphérie extrême, le paramètre du degré de proximité introduit une frontière essentielle entre les constituants, tant arguments que marges, qui se situent à l'intérieur du procès, et les marges qui se situent à l'extérieur. Le paramètre du régime de codage distingue les arguments codés en régime relationnel des arguments codés en régime ponctuel d'une part, et les marges codés en régime ponctuel des marges confiés à l'objet indirect d'autre part. Les trois paramètres sont indépendants : « interne " n'implique pas « contrôlé »; « non contrôlé » n'implique pas « externe»; " contrôlé » n’implique pas " codé en régime relationnel » et « non contrôlé » n'implique pas « codé en régime ponctuel ».

Grâce au paramètre du contrôle verbal, tous les arguments s'opposent à toutes les marges, et notamment tant aux circonstanciels, qui se situent à l'extérieur du procès, qu'aux modificateurs du verbes et aux marges du prédicat (M. Prandi, 1987, 2004), qui se situent à l'intérieur. Le paramètre du contrôle verbal annule tout espace logique pour imaginer tant une gradation de la catégorie d'argument qu'un continuum flou entre arguments et marges. Le même paramètre fait place aux adverbes et aux attributs essentiels à l'intégrité du procès qui, tout en n'étant pas des arguments, sont contrôlés par le verbe. La présence d'adverbes et d'attributs essentiels montre que la valence, définie comme le plan de fabrication d'un procès intègre dans sa structure conceptuelle, inclut le schéma d'arguments requis par le verbe mais ne s'y réduit pas nécessairement.

Le paramètre indépendant du régime de codage justifie la présence tant d'arguments qui partagent la forme d'expression des marges, que de marges qui par- 
tagent la forme d'expression des arguments, sans menacer pour autant la distinction essentielle entre arguments et marges.

Grâce au paramètre indépendant du degré de proximité, les modificateurs du verbe, les arguments et les marges du prédicat, qui se situent à l'intérieur du procès le long d'une échelle de proximité décroissante, s'opposent aux circonstanciels, qui lui sont externes. La progression logique dans l'association des différents constituants au pivot prédicatif et son indépendance du contrôle verbal peuvent être vérifiées à l'aide du critère de la mise en place progressive des conditions de cohérence des référents de chacun. Tout en n'étant pas contrôlé par le verbe, un modificateur s'y intègre logiquement avant l'objet direct : la preuve est qu'un modificateur du verbe est en mesure d'affecter la cohérence du référent de l'objet direct. Par exemple, on peut frapper tout objet concret, mais on ne peut frapper à mort qu'un être vivant : Le roc a frappé le serpent / le mur de la maison; Le roc a frappé à mort le serpent / *le mur de la maison. L'instrument, également, affecte le profil cohérent du sujet, ce qui implique qu'il s'intègre au procès après les arguments internes au prédicat mais avant le sujet : Pierre / Le roc a frappé le serpent; Pierre / 'Le roc a frappé le serpent avec un bâton. Le sujet, pour ainsi dire, scelle le procès, séparant les constituants internes des constituants externes.

Grâce à l'interaction des trois facteurs, chaque constituant trouve sa place exacte dans la structure du procès et la sensation de flou disparaît. Les arguments sont tous contrôlés indépendamment du régime de codage et les marges sont toutes non contrôlées indépendamment des différences dans le degré de proximité et dans le régime de codage. Comme un argument locatif codé en régime ponctuel n'est pas moins un argument qu'un agent confié au sujet, une marge interne confiée à l'objet indirect en présence d'un verbe divalent, et donc codé en régime relationnel, n'est pas moins une marge qu'un circonstanciel externe codé en régime ponctuel. Tout en étant essentiel à l'intégrité du procès, un adverbe ou un attribut n'est pas un argument; tout en n'étant pas un argument, il n'en est pas une marge pour autant.

Une fois que les distinctions entre arguments et marges, arguments et constituants essentiels différent des arguments, marges internes et externes, ont recouvré un profil clair et distinct, le seul obstacle que nous pouvons imaginer à une distinction tranchée est d'ordre factuel : nous ne pouvons pas exclure a priori que dans quelque cas isolé le statut exact d'un constituant spécifique dans une construction spécifique demeure indécidable. Même un cas pareil, cependant, ne serait pas une raison suffisante pour remettre en question les distinctions pertinentes. Le problème de la distinction entre arguments et marges, en effet, est transcendantal. Un problème est transcendantal quand les difficultés à trouver une solution ne remettent pas en question la structure du problème mais l'adéquation des instruments d'analyse. Le fait que ni la philosophie empiriste ni l'alternative rationaliste n'étaient en mesure d'expliciter les conditions de possibilité de l'entreprise scientifique à l'époque de Kant n'était pas une bonne raison pour nier son 
bien fondé. Le fait que l'on n'est pas capable de trouver l'assassin ne remet pas en question la réalité d'un meurtre. De la même façon, si les distinctions entre arguments et marges, arguments et constituants essentiels non argumentaux, marges internes et externes sont de nature conceptuelle, elles ne peuvent que s'imposer comme une nécessité logique par-delà les aléas des formes d'expression. La possibilité que les distinctions pertinentes demeurent quelque peu voilées pour nous qui les observons à travers la lentille de la forme d'expression, loin de remettre en question leur bien fondé, est une conséquence directe du fait que les formes de codage des arguments ne sont pas toujours univoques.

\section{Conclusions}

Les difficultés les plus significatives que l'on rencontre dans la description de la valence des verbes, et notamment dans la distinction entre arguments, adverbes et attributs essentiels, et marges d'une part, et dans la hiérarchisation des marges d'autre part, renvoient à une erreur méthodologique qui pousse à localiser les différences pertinentes directement dans la structure de l'expression. Comme nous l'avons constaté, les différences sont claires et distinctes au niveau d'une analyse conceptuelle fondée sur les critères de l'intégrité du procès et de la cohérence des marges mais se brouillent, faute de paramètres distinctifs univoques, au niveau des formes d'expression, qui entretiennent avec la structure conceptuelle du procès une relation indirecte à deux niveaux. En premier lieu, le décalage entre la phrase modèle et l'énoncé entraîne un glissement dans le critère de pertinence : si la phrase modèle a la fonction de garantir l'intégrité conceptuelle du procès, l'énoncé a la fonction d'intégrer un procès de façon cohérente dans le dynamisme communicatif d'un texte. Ensuite, les conditions de codage dans la phrase modèle offusquent la distinction principale entre arguments et marges du fait que certains arguments sont codés comme des marges et certaines marges sont codées comme des arguments. À ce point, si les stratifications complexes des formes d'expression, sur la base du présupposé que la forme d'expression reflète la structure conceptuelle, sont rétro-projetées sur la structure du procès, l'effet de flou est inévitable.

Pour sortir de l'impasse sans dissoudre l'idée même de valence dans l'enregistrement passif des configurations tendancielles documentées dans les corpus, il faut procéder en deux étapes.

Les distinctions pertinentes pour l'étude de la valence se fondent sur des critères qui sont en premier lieu conceptuels : les arguments, les adverbes et les attributs essentiels sont requis sur le plan conceptuel pour garantir l'intégrité du 
procès ; les marges sont des expansions optionnelles qui répondent à la condition de la cohérence conceptuelle, la même qui fonde leur hiérarchisation.

Une fois que la nécessité logique des distinctions est fondée sur des critères indépendants, le comportement des formes d'expression n'a plus le pouvoir de les remettre en question. L'analyse des structures syntaxiques, par conséquent, se pose comme objectif l'identification de corrélations significatives entre formes d'expression de la phrase modèle et constituants du procès. En régime de codage relationnel, la corrélation entre relations grammaticales et arguments est systématique à une exception près : il s'agit de l'objet indirect appliqué à des verbes divalents, qui code une famille de relations conceptuelles marginales. En régime de codage ponctuel, les propriétés formelles de l'expression ne sont pas en mesure de discriminer les arguments des marges confiées à la même forme d'expression. À ce point, l'objectif de sauvegarder la distinction pousse à identifier des critères à la fois conceptuels et textuels sensibles à la différence de fonction. L'utilisation conjointe des deux types de critères réduit le résidu indécidable à un seul cas l'interlocuteur d'un verbe de communication symétrique comme discuter - qui peut être réglé avec le critère qui oppose le contrôle verbal sur le contenu des arguments à l'accessibilité par inférence du contenu des marges.

Suite à l'application de critères différenciés et adéquats à la structure du problème, les distinctions pertinentes entre arguments et éléments contrôlés non argumentaux, arguments et marges, marges externes et marges internes apparaissent comme des différences multifactorielles. Si nous considérons l'action conjointe de tous les paramètres en interaction, à savoir le contrôle verbal, le degré de proximité et le régime de codage, le risque de flou se dissout, et la configuration qui apparaît comme un continuum se révèle être une hiérarchie de relations multifactorielles.

\section{Références citées}

Blinkenberg, A. (1960). Le problème de la transitivité en français moderne : essai syntacto-sémantique. København, I Kommission hos Munksgaard.

Chomsky, N. A. (1971 [1965]). Aspects of the Theory of Syntax. Cambridge (Massachusetts), MIT Press [Traduction française : Aspects de la théorie syntaxique (J.-Cl. Milner, Trad.). Paris, Seuil].

Dowty, D. (2000). The dual analysis of adjuncts / complements in categorial grammar. ZAS Papers in Linguistics, 17, 1-26. Réimpr. in E. Lang, I. Maienbom \& C. Fabricius-Hansen (Eds.), Modifying Adjuncts (p. 33-66). Berlin — New York, De Gruyter Mouton.

Feuillet, J. (Éd.). (1998). Actance et Valence dans les Langues de l'Europe. Berlin, De Gruyter Mouton. 
Firbas, J. (1964). On defining the theme in functional sentence analysis. Travaux Linguistiques de Prague, I, 267-80.

Goldberg, A. E. (1995). Constructions. A Construction Grammar Approach to Argument Structure. Chicago - London, University of Chicago Press.

Gross, G. (2012). Manuel d'analyse linguistique. Villeneuve-d'Ascq, Presses universitaires du Septentrion.

Gross, G., \& Prandi, M. (2004). La finalité : fondements conceptuels et genèse linguistique. Bruxelles, De Boeck / Duculot.

Halliday, M. A. K. (1970). Linguistic Structure and Linguistic Function. In J. Lyons (Ed.), New Horizons in Linguistics (p. 140-165). Harmondsworth, Penguin Books.

Hanks, P. (2013). Lexical Analysis. Cambridge (Massachusetts), MIT Press.

Hilpert, M. (2014). Construction Grammar and its Application to English. Edinburgh, Edinburgh University Press.

König, E., \& Haspelmath, M. (1998). Les constructions à possesseur externe dans les langues de l'Europe. In J. Feuillet (Éd.), Actance et Valence dans les Langues de l'Europe (p. 525-606). Berlin, De Gruyter Mouton.

Lazard, G. (1998). Définition des actants dans les langues européennes. In J. Feuillet (Éd.), Actance et Valence dans les Langues de l'Europe (p. 111-146). Berlin, De Gruyter Mouton.

Longacre, R. E. (2006 [1985]). Sentences as Combinations of Clauses. In T. Shopen (Ed.), Language Typology and Syntactic Description (2nd ed., Vol. II : Complex Constructions, p. 235-286). Cambridge, Cambridge University Press.

Lyons, J. (1977). Semantics. Cambridge, Cambridge University Press.

Mereu, L. (2017). La struttura argomentale in un approccio 'usage-based'. Studi e saggi linguistici, 55(2), 36-65.

Mereu, L. (2020). La semantica della frase. Roma, Carocci.

Mereu, L., \& Piunno, V. (2019). The Argument Structure of Verbs of Hitting and Breaking in Italian. Lingue e linguaggio, 18(1), 143-176.

Orlandi, A., \& Fasciolo, M. (2021). Corpus Pattern Analysis et Classes d'objets : différences théoriques et retombées pratiques de deux approches à la description du lexique. Synergies Italie, 17, 91-105.

Palmer, F. R. (1994). Grammatical Roles and Relations. Cambridge, Cambridge University Press.

Perek, F. (2015). Argument Structure in Usage-Based Construction Grammar. Amsterdam — Philadelphia, John Benjamins.

Prandi, M. (1987). Sémantique du contresens. Paris, Éditions de Minuit.

Prandi, M. (2004). The Building Blocks of Meaning. Ideas for a Philosophical Grammar. Amsterdam — Philadelphia, John Benjamins.

Prandi, M. (2019). Phrase et énoncé : de l'ordre symbolique à l'ordre indexical. In F. Neveu (Éd.), Proposition, phrase, énoncé. Linguistique et philosophie (p. 131-154). Londres, Iste Editions.

Prandi, M. (2020). Roles and grammatical relations in synchrony and diachrony: the case of the indirect object. In C. Fedriani \& M. Napoli (Eds.), The Diachrony of Ditransitives (p. 19-58). Berlin, De Gruyter Mouton.

Pustejowsky, J. (1995). The generative Lexicon. Cambridge (Massachusetts), MIT Press. 
Pustejovsky, J. (2000). Lexical shadowing and Argument Closure. In Y. Ravin \& C. Leacock (Eds.), Polysemy. Theoretical and Computational Approaches (p. 68-90). Oxford, Oxford University Press.

Riegel, M., Pellat, J.-Ch., \& Rioul, R. (1994). Grammaire méthodique du français. Paris, Presses universitaires de France.

Steinitz, R. (1969). Adverbial-Syntax. Berlin, Akademie Verlag.

Strik-Lievers, F. (2012). Sembra ma non è. Studio semantico-lessicale sui verbi con complemento predicativo. Firenze, Accademia della Crusca.

Tesnière, L. (1965 [1959]). Éléments de syntaxe structurale ( $2^{\mathrm{e}}$ éd.). Paris, Klincksieck.

Vergnaud, J.-R., \& Zubizarreta, M.-L. (1992). The definite determiner and the inalienable construction in French and English. Linguistic Inquiry, 23, 595-652. 